\title{
Utilising Electronic Health Record Data to Assess the Sepsis Inpatient Care Pathway: A Feasibility Study
}

\author{
Ling LI ${ }^{\mathrm{a}, 1}$, Kasun RATHNAYAKE ${ }^{\text {a }}$, Tsui Yue ONG ${ }^{\mathrm{b}}$, Cliff HUGHES ${ }^{\mathrm{c}}$, \\ Vincent LAM $^{\mathrm{c}}$ and Johanna I WESTBROOK ${ }^{\mathrm{a}}$ \\ ${ }^{a}$ Centre for Health Systems \& Safety Research, Macquarie University, Australia \\ ${ }^{b}$ Nepean Hospital, Penrith, New South Wales, Australia \\ ${ }^{c}$ Department of Clinical Medicine, Macquarie University, Australia
}

\begin{abstract}
The World Health Organisation has recently declared sepsis a global medical emergency. Obtaining quality data to establish the evidence on how clinicians recognise, diagnose, and treat sepsis is still a challenge. This feasibility study aimed to utilise routinely collected data from electronic health records (EHR) to assess the sepsis inpatient care pathway. We conducted a retrospective observational cohort study which included all patients admitted to a private teaching hospital between 2015 and 2018. De-identified patient demographic and clinical data were extracted and analysed. A total of 47 sepsis patients were identified based on diagnoses recorded and a review of clinical notes. A surgical procedure was conducted on more than half of these patients $(\mathrm{n}=25,53 \%)$. Nearly two-thirds were given antibiotics $(\mathrm{n}=30,64 \%)$, of which $87 \%(\mathrm{n}=26)$ were administered within 2hours of sepsis diagnosis. Eighteen patients were admitted to ICU and 13 of them were diagnosed as septic in ICU. We identified some aspects of EHR data that could be improved. Overall, routinely collected data from clinical information systems provides rich information to assess the sepsis patient care pathway.
\end{abstract}

Keywords. sepsis, patient care pathway, electronic health record, antibiotics, sepsis treatment

\section{Introduction}

Sepsis is defined as "life-threatening organ dysfunction caused by a dysregulated host response to infection" [1]. Despite medical advances, it remains a major cause of morbidity and mortality worldwide. The most recent global burden of disease study, published in the Lancet, reported that 48.9 million new cases of sepsis were recorded worldwide in 2017 with 11 million sepsis-related deaths, representing $20 \%$ of all deaths globally [2]. The first national sepsis epidemiology report shows that motality rate of sepsis patients is 11 times higher than that of non-sepsis patients in Australian hospitals [3]. Addressing the challenge, the World Health Assembly of the World Health Organisation (WHO) passed a resolution in 2017 on better prevention, diagnosis, and

\footnotetext{
${ }^{1}$ Corresponding Author, Associate Professor Ling Li, Centre for Health Systems and Safety Research, Australian Institute of Health Innovation, Macquarie University, NSW 2109, Australia. Email: ling.li@mq.edu.au.
} 
management of sepsis [4]. Obtaining quality data to assess how health systems perform in diagnosing and managing sepsis cases remains challenging.

The ongoing rollout of electronic health records (EHR) across modern healthcare systems presents unprecedented opportunities to harness large volumes of data for analysis to answer important health questions. EHRs contain information such as patient's diagnoses, medications taken, and laboratory test results. EHR data has been used to improve the performance of clinical decision support systems for early detection of sepsis [5]. This feasibility study aimed to utilise datasets extracted from different clinical information systems in an EHR to assess the sepsis inpatient care pathway and identify the potential limitations of EHR data.

\section{Method}

\subsection{Study Design, Setting and Population}

We conducted a retrospective observational study undertaken at a 180-bed private teaching hospital in Sydney, Australia. Pathology, radiotherapy and imaging are available on site. All adult patients (age $\geq 18$ years) admitted to the hospital from 2015 to 2018 were included. Ethics approval for this study was granted by the Macquarie University Human Research Ethics Committee (Reference No: 5201600379).

\subsection{Data Sources and Linkage}

Patient demographic data (e.g. age and sex), as well as admission and clinical characteristics (e.g. medications taken, laboratory test results, surgical procedures, diagnoses, and ICU admissions) were extracted from the hospital's clinical and administrative information system. Data sets from different systems were merged using de-identified unique patient identifier and time stamp data where relevant.

\subsection{Identifying Patients with Sepsis and Diagnosis Time}

Patients with sepsis were identified based on: i) International Statistical Classification of Diseases and Related Health Problems, tenth revision, Australian modification (ICD-10AM) sepsis-related codes as defined by the Classification of Hospital Acquired Diagnoses approach [6], and ii) the review of selected clinical notes from patient admissions by a medical staff (see Figure 1). The clinical notes were selected if keywords related to sepsis or infection were recorded by clinicians, e.g. sepsis, septic, septic shock, infection, inflammation, febrile, high temperature, blood culture, culture, specimen, bacilli, gram, de-saturating, and pneumonia. This review process identified additional sepsis patients without ICD-10-AM codes recorded. The reviewer also recorded the sepsis diagnosis time if available in the clinical notes. All available clinical information related to these sepsis patient admissions were then merged to map out the patient care pathway during hospital stays. This information included any ICU admissions, ward transfers, surgical procedures performed, antibiotics administered, blood cultures ordered, and clinical measurements. 


\subsection{Data Management and Analysis}

Data quality was assessed across six dimensions [7]. To ensure completeness of the data regarding sepsis patient identification and time of sepsis diagnosis, we conducted text mining and review of clinical notes in addition to diagnoses recorded (as stated in the previous section). The data were likewise checked for the remaining five data quality dimensions: timeliness (represents reality from the required point in time), uniqueness (nothing is recorded more than once based upon how that thing is identified), validity (conforms to the syntax format, type, and range), accuracy (correctly describes the "real world" object or event being described) and consistency (agrees across different data sets, and the extent of agreement between different data sets that are measuring the same thing).

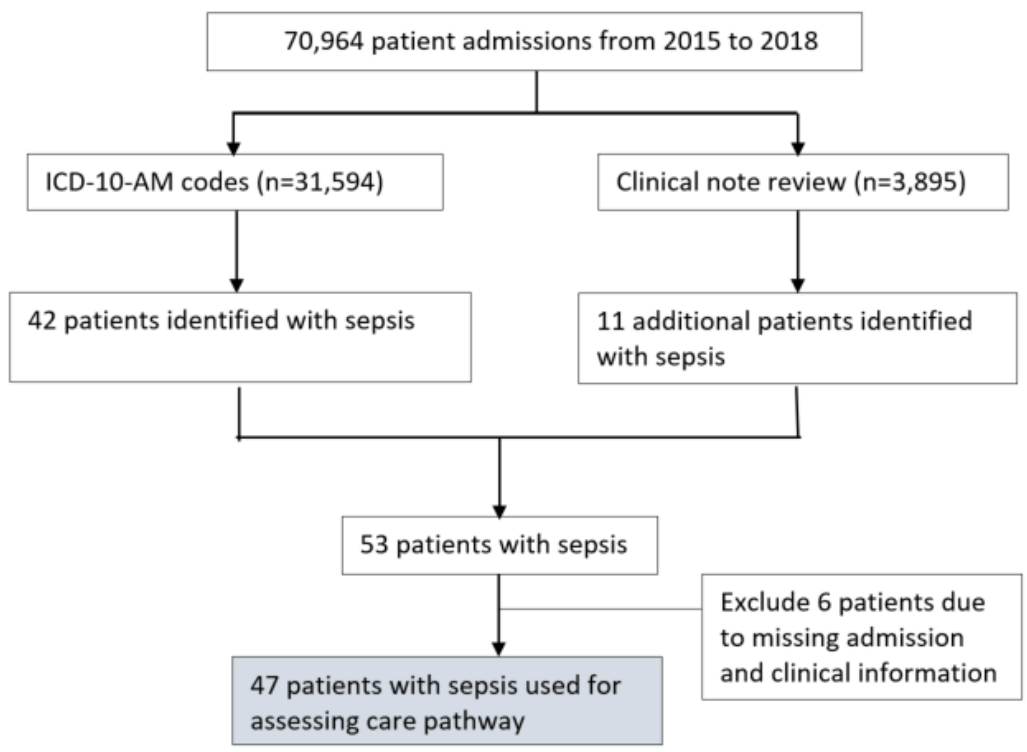

Figure 1. Flow diagram of study population and sepsis cases identification

Descriptive summary statistics were presented to summarise the sepsis patient care pathway data. Sepsis diagnosis dates and times, identified by the reviewer from clinical notes, were used to further examine the sepsis management process in conjunction with other timing information, such as the timing of surgical procedures, ICU admissions, and antibiotic administration. The data management and analysis were conducted using $\mathrm{R}$, version 3.6.1.

\section{Results}

\subsection{Sepsis Patients}

There were 70,964 patient admissions during the study period. Patient demographic information (e.g. age and sex), were consistently completed. Clinical information was recorded for patients, including 43,964 admissions with medications administered, 
14,356 admissions with laboratory testing results, 21,142 admissions with clinical procedures (e.g. surgery) and 31,594 admissions with ICD-10-AM codes. A total of 53 sepsis patients were identified: 42 through ICD-10-AM coding recorded in patients' diagnoses and 11 based on clinical notes review (Figure 1). Six were excluded from further analysis due to missing admission and clinical information, leaving a final cohort of 47 sepsis patients.

The mean age of these 47 patients was 67 years, and 29 patients were male $(62 \%)$. Two patients died during hospitalisation. The most common comorbidities recorded were hypertension $(n=13,28 \%)$, urinary tract infection $(n=11,23 \%)$ and hypotension $(\mathrm{n}=10,21 \%)$. The median length of stay was 9.5 days (Inter-quartile range- IQR: 2.1 33.4 days).

More than one half of these patients underwent a surgical procedure during their hospital stay ( $n=25,53 \%$; Figure 2). Of these surgical patients, time of sepsis diagnosis was identified for 18 patients. The median time from surgical procedure to diagnosis of sepsis was 3.4 hours (IQR: 0.9-21.1 hours).

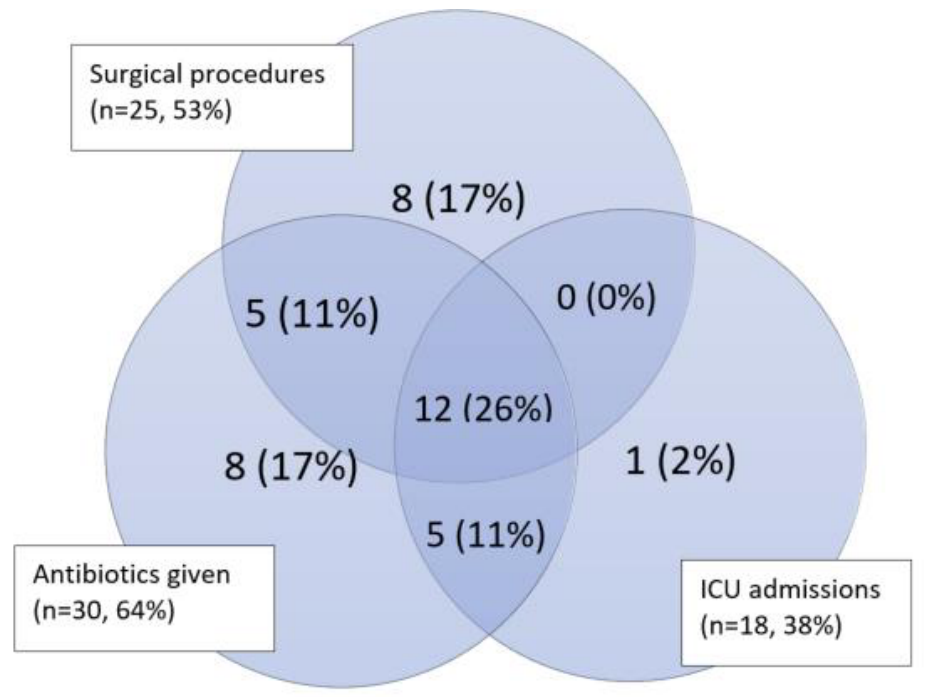

Figure 2. Sepsis patients who had a surgery, antibiotics, an ICU admission, or combination of these factors while in hospital (percentages based on the total sample of 47 sepsis patients)

\subsection{Sepsis Patient Care and Management}

Eighteen sepsis patients (38\%) were admitted to ICU (Figure 2). Among them, 13 of these patients were diagnosed with sepsis during their ICU stay. The median length of ICU stay was 4.8 days (IQR: 2.0-11.2 days). Only one of these 18 patients had no antibiotic administration recorded.

Nearly two-thirds of the 47 patients had antibiotics administered ( $n=30,64 \%)$. Among them, 28 patients were given antibiotics after sepsis diagnosis: 6 patients within 1-hour of diagnosis and 20 within 1-2 hours. The most common antibiotics used for these patients were: Cephazolin, Vancomycin, Ceftriaxone, Piperacillin, Ciprofloxacin IV, and Dexamethasone. On reviewing sepsis-related laboratory tests, 10 patients had blood 
culture and other culture tests ordered prior to antibiotic administration, 26 had creatinine tests, 18 had C-reactive protein tests (CRP), and only two had lactate ordered.

\section{Discussion}

A total of 70,964 patient admissions were included in this feasibility study to identify sepsis patients and describe their care pathways. We retrospectively examined a large volume of routinely collected data from different clinical information systems and found the data sets contained incomplete information in key areas. Firstly, sepsis diagnoses were not recorded for 11 patients, representing $21 \%$ of the total 53 sepsis patients identified. These 11 patients were identified through retrospective review of clinical notes. Secondly, this review process identified another critical piece of missing information: the timing of sepsis diagnosis. In addition, six sepsis patients had missing admission and other clinical information Despite these limitations, the EHR data provided extensive information about sepsis patients and their hospital stay, including type and time of surgical procedures, laboratory testing, and antibiotic administration.

Numerous global, national, and local, clinical guidelines have been developed to support sepsis recognition, diagnosis, and early treatment. Internationally, the latest 2017 Surviving Sepsis Guideline panel provided 93 statements on early management and resuscitation of patients with sepsis or septic shock [8]. In Australia, the prominent SEPSIS KILLS program provides sepsis care pathways and guidelines widely used in NSW public hospitals [9]. Our study found that routinely collected EHR data, includes time stamped records of important patient hospital stay information, making it possible to assess patient care, and patient care timelines, against these established clinical guidelines. Antibiotic administration is one of the most commonly recommended sepsis treatments. In our study cohort, antibiotics were administered quickly when given, as per guidelines, with 26 out of 30 patients being given antibiotics within 2 hours of diagnosis. However, the remaining 17 out of 47 sepsis patients were not recorded to have been given antibiotics. Moreover, laboratory tests, including blood culture, are recommended prior to administrating antibiotics. In this study, we found only 10 out of 47 patients had blood culture and other culture tests ordered prior to antibiotic administration.

While much attention has been placed on the identification of sepsis in ICU patients, we found only 10 out of 47 patients were diagnosed while in ICU, consistent with findings from previous studies $[10 ; 11]$. Previous studies also highlight the relatively high sepsis mortality rates among the general ward patients, up to $50 \%$ [10-12]. Thus, further investigation on the diagnosis and treatment of sepsis in general ward patients is justified. More than half the patients in our study had surgery, which is similarly in line with results from other studies [13].

This feasibility study was conducted at a private teaching hospital, which does not have an emergency department and does not offer a comprehensive range of hospital care services. Therefore, the reported incidence of sepsis might be lower than the average general teaching hospital. Additionally, while the small number of sepsis patients made elements of our study easier to manage, such as conducting chart review $(n=3,895$ patients), caution should be taken when generalising these results.

Overall, findings from this feasibility study warrant further investigation using a larger population to conduct a comprehensive analysis of the sepsis care pathway after addressing the current limitations of EHR data. Quality EHR data will not only help us to understand the variations in sepsis care against relevant clinical guidelines, but also 
provide a foundation for designing and implementing an electronic sepsis care pathway to improve sepsis patient care and outcomes.

\section{Conclusion}

We found that routinely collected data from clinical information systems provided rich information to assess the sepsis patient care pathway. We identified some aspects of EHR data that could be improved to enhance the use of such quality data for comparing sepsis care against current clinical guidelines and for designing and implementing an electronic sepsis care pathway to improve patient outcomes.

\section{References}

[1] M. Singer, C.S. Deutschman, C.W. Seymour, M. Shankar-Hari, D. Annane, M. Bauer, R. Bellomo, G.R. Bernard, J.D. Chiche, C.M. Coopersmith, R.S. Hotchkiss, M.M. Levy, J.C. Marshall, G.S. Martin, S.M. Opal, G.D. Rubenfeld, T. van der Poll, J.L. Vincent, and D.C. Angus, The Third International Consensus Definitions for Sepsis and Septic Shock (Sepsis-3), JAMA 315 (2016), 801-810.

[2] K.E. Rudd, S.C. Johnson, K.M. Agesa, K.A. Shackelford, D. Tsoi, D.R. Kievlan, D.V. Colombara, K.S. Ikuta, N. Kissoon, S. Finfer, C. Fleischmann-Struzek, F.R. Machado, K.K. Reinhart, K. Rowan, C.W. Seymour, R.S. Watson, T.E. West, F. Marinho, S.I. Hay, R. Lozano, A.D. Lopez, D.C. Angus, C.J.L. Murray, and M. Naghavi, Global, regional, and national sepsis incidence and mortality, 1990-2017: analysis for the Global Burden of Disease Study, The Lancet 395 (2020), 200-211.

[3] L. Li, N. Sunderland, K. Rathnayake, and J. Westbrook, Epidemiology of Sepsis in Australian Public Hospitals: A Mixed Methods, National Longitudinal Study (2013-2018), Australian Commission on Safety and Quality in Health Care, 2020.

[4] World Health Organisation, Service delivery and safety. Improving the prevention, diagnosis and clinical management of sepsis, retrieved from https://www.who.int/servicedeliverysafety/areas/sepsis/en/.

[5] L. Li, K. Rathnayake, M. Green, M. Fullick, A. Shetty, S. Walter, J. Braithwaite, H. Lander, and J.I. Westbrook, Improving the Performance of Clinical Decision Support for Early Detection of Sepsis: A Retrospective Observational Cohort Study, Stud Health Technol Inform 264 (2019), 679-683.

[6] T.J. Jackson, J.L. Michel, R.F. Roberts, C.M. Jorm, and J.G. Wakefield, A classification of hospitalacquired diagnoses for use with routine hospital data, Medical Journal of Australia 191 (2009), 544-548.

[7] Data Quality Dimentaions Working Group, The Six Dimensions of EHDI Data Quality Assessment, DAMA UK, 2013.

[8] A. Rhodes, L.E. Evans, W. Alhazzani, M.M. Levy, M. Antonelli, R. Ferrer, A. Kumar, J.E. Sevransky, C.L. Sprung, M.E. Nunnally, B. Rochwerg, G.D. Rubenfeld, D.C. Angus, D. Annane, R.J. Beale, G.J. Bellinghan, G.R. Bernard, J.D. Chiche, C. Coopersmith, D.P. De Backer, C.J. French, S. Fujishima, H. Gerlach, J.L. Hidalgo, S.M. Hollenberg, A.E. Jones, D.R. Karnad, R.M. Kleinpell, Y. Koh, T.C. Lisboa, F.R. Machado, J.J. Marini, J.C. Marshall, J.E. Mazuski, L.A. McIntyre, A.S. McLean, S. Mehta, R.P. Moreno, J. Myburgh, P. Navalesi, O. Nishida, T.M. Osborn, A. Perner, C.M. Plunkett, M. Ranieri, C.A. Schorr, M.A. Seckel, C.W. Seymour, L. Shieh, K.A. Shukri, S.Q. Simpson, M. Singer, B.T. Thompson, S.R. Townsend, T. Van der Poll, J.L. Vincent, W.J. Wiersinga, J.L. Zimmerman, and R.P. Dellinger, Surviving Sepsis Campaign: International Guidelines for Management of Sepsis and Septic Shock: 2016, Intensive Care Med 43 (2017), 304-377.

[9] Clinical Excellence Commission, SEPSIS KILLS - Adult Sepsis Pathway, retrieved from http://www.cec.health.nsw.gov.au/_ data/assets/pdf_file/0005/291803/Adult-Sepsis-Pathway-Sept2016-with-watermark.pdf.

[10] A. Esteban, F. Frutos-Vivar, N.D. Ferguson, O. Penuelas, J.A. Lorente, F. Gordo, T. Honrubia, A. Algora, A. Bustos, G. Garcia, I.R. Diaz-Reganon, and R.R. de Luna, Sepsis incidence and outcome: contrasting the intensive care unit with the hospital ward, Crit Care Med 35 (2007), 1284-1289.

[11] J.M. Rohde, A.J. Odden, C. Bonham, L. Kuhn, P.N. Malani, L.M. Chen, S.A. Flanders, and T.J. Iwashyna, The epidemiology of acute organ system dysfunction from severe sepsis outside of the intensive care unit, J Hosp Med 8 (2013), 243-247.

[12] L. Li, K. Rathnayake, M. Green, A. Shetty, M. Fullick, S. Walter, C. Middleton-Rennie, M. Meller, J. Braithwaite, H. Lander, and J.I. Westbrook, Comparison of the Quick Sepsis-Related Organ Failure 
Assessment (qSOFA) and Adult Sepsis Pathway in Predicting Adverse Outcomes among Adult Patients on General Wards: A Retrospective Observational Cohort Study, Internal Medicine Journal 10.1111/imj.14746.

[13] [13] A.C.G.P. Elias, T. Matsuo, C.M.C. Grion, L.T.Q. Cardoso, and P.H. Verri, Incidence and risk factors for sepsis in surgical patients: A cohort study, Journal of Critical Care 27 (2012), 159-166. 